\title{
Nonlinear transverse steady-state periodic forced vibration of 2-dof discrete systems with cubic nonlinearities
}

\author{
Ahmed Eddanguir* — Zitouni Beidouri** — Rhali Benamar* \\ * LERSIM, Ecole Mohammadia d'Ingénieurs Rabat \\ Université Mohammed $V$ \\ Avenue Ibn Sina, Agdal, Rabat, Maroc \\ eahmed66@hotmail.com ; rbenamar@emi.ac.ma \\ ** LMPGI, Ecole Supérieure de Technologie de Casablanca \\ Université Hassan II \\ Ain Chock, Km 7 Route El Jadida, Casablanca, Maroc \\ zbeidouri@gmail.com
}

\begin{abstract}
A method based on Hamilton's principle and spectral analysis has been applied recently to nonlinear transverse vibrations of discrete systems with cubic nonlinearities, leading to calculation of the nonlinear free modes of transverse vibration and their associated nonlinear frequencies. The objective of the present work was the extension of this method to the nonlinear forced transverse steady-state periodic response of 2-dof system leading to nonlinear frequency response function in the neighbourhood of the two modes

RÉSUMÉ. La méthode basée sur le principe d'Hamilton et l'analyse spectrale a été récemment appliquée aux vibrations libres transversales non linéaires des systèmes discrets à nonlinéarités cubiques, conduisant au calcul de modes propres non linéaires des vibrations transversales et des fréquences non linéaires associées en fonction de l'amplitude de vibration. L'objectif de cet article est l'extension de cette méthode aux vibrations transversales non linéaires forcées des systèmes discrets soumis à des forces périodiques et d'avoir la réponse fréquentielle non linéaire au voisinage des deux modes non linéaires, par voie analytique et numérique.

KEYWORDS: nonlinear transverse vibration, 2-dof system, Hamilton's principle, explicit procedure, steady-state periodic forced response.

MOTS-CLÉS : vibration transversale non linéaire, système à 2 ddl, principe d'Hamilton, procédure explicite, réponse périodique forcée en régime permanent.
\end{abstract}

DOI:10.3166/EJCM.20.143-166 @ 2011 Lavoisier, Paris

EJCM - 20/2011. Dynamics of materials, structures and systems, pages 143 to 166 


\section{Introduction}

The objective of the present work is the treatment of the transverse steady-state periodic forced response of 2-dof systems with cubic nonlinearities, using the method developed in references (Eddanguir et al., 2009; Beidouri et al., 2008; El Kadiri et al., 2002b; Benamar et al., 1993) and applied to the investigation of nonlinear vibration of different structures, of various types, geometries, and material characteristics.

In a nonlinear system, the relationship between cause and effect is no longer proportional, which has very important consequences, both quantitative and qualitative, on the dynamic behaviour. Due to this important property of nonlinear systems, the nonlinear behaviour exhibits many complicating aspects, such as the uniqueness and existence of solutions which are not always guaranteed, the existence of instable regions at which the jump phenomena may occur, the curvature of the frequency response functions to the left or to the right, depending on the type of nonlinearity, i.e. softening or hardening, the amplitude dependence of the system stiffness, and consequently, of the mode shapes and resonant frequencies; the presence of many harmonics in the nonlinear free or forced response, the occurrence of chaotic behaviour (see for instence (Azrar, 1999; Benamar, 1990; Bennouna, 1982; White, 1971)).

Also, it should be noticed that vibrating systems may exhibit sometimes, in addition to the distributed nonlinearity, various kinds of localised nonlinearity. This is the case for example of a beam with a non-uniform cross section, a plate with stiffeners, or when the beam or the plate carries a concentrated mass, or rests on a nonlinear spring at a given point. In such cases, discretised models, such as that presented here, may be very useful for approaching, with a reasonable computational effort, the nonlinear behaviour.

The purpose of the present work is determination of the nonlinear frequency response function of a 2-dof system using an approximate explicit method in the neighbourhood of the two resonant frequencies.

The next step following the present work is the extension of this method to the nonlinear transverse steady-state periodic forced response of $\mathrm{N}$-dof systems and the application of the discrete model developed to study nonlinear transverse vibration of beams having localised nonlinearities, due, for example to discontinuities in the mass or in the rigidity distributions.

\section{Brief state of the art on the subject}

Without attempting a comprehensive review, which would exceed the scope of the present paper, some of the works related to the subject of nonlinear structural vibrations are going to be mentioned and briefly commented below : 


\subsection{Brief review on discrete systems}

The notion of normal modes of nonlinear Multi-degree of freedom systems was introduced by Rosenberg in the beginning of the sixties (Rosenberg, 1962). The work of Rosenberg was the foundation of most of what followed. The system considered was constituted by $\mathrm{N}$-masses interconnected by nonlinear springs, and the first and last of the masses were connected by nonlinear springs to fixed points (or to infinitely large masses). The system was supposed to be conservative and the equations of motion were derived from a potential. The following definition of the nonlinear normal modes was based on the observation that the normal solutions of the linear system are all periodic of the same period, and that the ratio of the displacement of any mass to that of any other is identically equal to a constant for all time : "The nonlinear system is said to be vibrating in normal modes when :

- All masses vibrate at the same frequency.

- They pass through their equilibrium position at the same time.

- When, at any time $t$, the position of all the masses is uniquely defined by the position of any one of them".

The concept of 'Nonlinear Normal Mode' (NNM) has been discussed by Vakakis in (Vakakis, 1997) and used to study forced resonances of nonlinear systems, and nonlinear localisation of vibrational energy in symmetric systems. Additional applications of NNMs to modal analysis, model reduction, vibration and shock isolation designs, and the theory of nonlinear oscillators were also discussed. In reference (Lamarque, 2000), the study was restricted to simple and two degree of freedom systems, with a unilateral constraint on one of the degrees of freedom, for which the response can be analytically determined. Generalized frequencies, modes and masses were built in the procedure. The results obtained for various sets of parameters indicated some limitations to the validity of a general modal superposition formula. Reference (Chakraborty et al., 2001), was concerned with the dynamics of a weakly nonlinear periodic chain. This periodic structure is constituted by repeating identical systems which are called elements. Such a discrete structure without ends is called a cyclic periodic structure. Harmonic wave propagation in an infinite, nonlinear periodic chain was investigated. The nonlinear normal modes found consisted of almost two linear modes, and for some boundary conditions, exhibited restricted orthogonality properties. Some explicit numerical results were included to validate the wavepropagation approach for studying free vibration of such nonlinear periodic chains.

The two dof systems have been treated in several papers to illustrate the validity and efficiency of the methods developed for discrete systems in thesis works. Then, in reference (Lewandowsky, 2003), a symmetric 2-dof system has been treated by R. Lewandowsky using the classical Galerkin's method to give some remarks on what he called Benamar's method. This investigator has not succeded in his attempt to apply the method, mainly because of the necessity of writing the amplitude equation in the modale basis in order to apply properly the method. Due to this misunderstanding, we have been leaded to develop for the first time the theory for discrete systems at large 
vibration amplitudes using Benamar's method. Other works have been made in this field in (Pesheck et al., 2002; Shaw et al., 1993), based on a new Galerkin's approach using the invariant manifolds for accurate nonlinear normal modes of discrete systems. This approach was extended to nonlinear normal modes under harmonic excitation in the reference (Jiang et al., 2005), leading to determination of the frequency response for a simple 2-dof mass-spring system with cubic nonlinearities and for a discretized beam model with 12-dof. N-dof systems are still in interest and lead to several works by physician and mathematician investigators using different methods and taking into account several conditions : linear or nonlinear, free or forced, damped or un-damped. A new concept to derive upper bounds of the solution of initial value problems for free linear and nonlinear ordinary differential equations representing vibration problems was presented in reference (Kohaupt, 2004).

\subsection{Brief review on the work on nonlinear finite element method}

As mentioned above, the difficulties involved in nonlinear problems have led investigators to concentrate their effort on the understanding of the nonlinear behaviour associated with simple geometries, such as beams, plates and shells. However, some effort has been devoted in parallel to develop adequate nonlinear finite element formulations in order to provide engineers and analysts with adequate tools for investigating the behaviour of complex structures. There were some pioneer investigators which have developed the finite element method for nonlinear vibration problems such as in references (Mei, 1986; Mei, 1985; Mei, 1984; Mei, 1973), the finite element displacement method for large amplitude free flexural vibrations of beams and plates and forced vibrations of rectangular plates was investigated, and also comments and discussions on "Lagrange-type formulation for finite element analysis of nonlinear beam vibrations" were made.

More recently, new developments in the finite element method and the considerable interest in composite materials induced many papers on the linear vibrations of laminated rectangular plates in references (Han et al., 1996b; Han et al., 1996a). The first reference was concerned by free linear vibration analysis of symmetrically laminated, rectangular plates with clamped boundary conditions. It was shown that for hierarchical finite element method that the natural frequencies converge rapidly with an increase in the number of polynomials used, resulting in far fewer degrees of freedom than those required when using a conventional finite element method. It was shown that the mode shapes of symmetrically laminated plates depend upon the degree of bending-twisting coupling. The linear forced vibration of isotropic and symmetrically laminated rectangular plates was studied in the second reference by using the hierarchical finite element method. The loads considered have been harmonic acoustic plane waves impinging on the plate surface in a normal direction and at grazing incidence. It was found that with far fewer degrees-of-freedom than conventional finite element method, accurate results may be produced using the HFEM. The autors of references (Ribero et al., 1999a; Ribero et al., 1999b; Ribero, 1999a; Ribero, 1999b) have conti- 
nued in the field of finite element method applied to nonlinear vibration problems by studying the model for geometrical nonlinear, steady, forced and periodic vibration of plates based on hierarchical finite element method, and the stability analysis of multimodal response. Composite laminated plates and beams with internal resonance have also been considered.

\subsection{Presentation and discussion of The Benamar's method}

The so-called Benamar's method in reference (Lewandowsky, 2003) is based on a theoretical model based on spectral analysis, using the linear mode shapes of the structure considered as a basis for the expansion of the unknown displacement series. It reduces the large vibration amplitude problem, via use of Hamilton's principle and spectral analysis, and integration of the time function over a period of vibration, to a set of nonlinear algebraic equations which is solved numerically for each value of the amplitude of vibration. This method has been applied to a variety of situations, which can be classified as follows :

- Different structures : beams, plates, shells (Beidouri et al., 2006; Moussaoui et al., 2000; Azrar et al., 1999a; Benamar et al., 1994; Benamar et al., 1991; Benamar, 1990).

- Different geometries : rectangular and circular plates, finite and infinite shells, rings (see for instence the just cited referenceses and also (Rougui et al., 2003; Haterbouch et al., 2003; Moussaoui et al., 2001)).

- Different end conditions of beams and edge conditions of plates (see for instence previous references from (El Bikri et al., 2003) in addition to (Benamar et al., 1994)).

- Different material laws : isotropic, composites (see references cited above with (Haterbouch et al., 2005; Harras et al., 2002))

- Different types of problems : nonlinear free response, nonlinear forced response (references cited above in addition to (Beidouri et al., 2006; Azrar et al., 2002; Azrar et al., 1999b)).

- Several description levels :

- Assuming a harmonic motion, or assuming in addition to the harmonic response a harmonic distortion of the response (Atmani, 2005; Benamar, 1990).

- Neglecting the in-plane displacements in the modelling of nonlinear transverse vibrations of plates, or including these displacements in the theory (Haterbouch, 2004).

- To continuous structures and discrete systems (see for example (Eddanguir et al., 2009; Beidouri et al., 2008; Haterbouch, 2004; El Bikri et al., 2006; El Kadiri et al., 2002a; Azrar, 1999; Benamar et al., 1994)).

- Different methods of solution : iterative, explicit and linearized (see for example (El Bikri et al., 2006; Atmani et al., 2004; Haterbouch et al., 2004; Haterbouch, 2004; El Kadiri et al., 2002a; Moussaoui et al., 2002)). 
Two main features of this method could be deduced from the above mentioned works as follows :

a- In all of the problems considered, the nonlinear vibration problem is presented by three nonlinear dynamic characteristics, i.e. the mass tensor, the rigidity tensor, and the nonlinear rigidity tensor. The last tensor is most of the times a fourth order tensor. The formulation reduces to the classical modal analysis theory when the nonlinear effects are neglected.

b- The nonlinear vibration problem is presented by a nonlinear algebraic system, or a nonlinear eigenvalue problem, which reduces to the classical linear eigenvalue problem, very common and well known in the linear modal analysis theory.

c- The solution process, conducted by any mean, i.e. iterative, linearised or explicit, leads in all cases to a set of amplitude dependent mode shapes, i.e. the so-called nonlinear mode shapes, with the associated amplitude dependent nonlinear frequencies.

d- The experience accumulated in solving iteratively the nonlinear eigen value problems have conducted members of the nonlinear dynamic group team to notice that explicit solution of such problems can be obtained by rewriting the system in the modal basis, i.e. the basis of the system linear mode shapes, and making appropriate approximations. For very high amplitudes, for which the approximations made do not work any more, another approach has been developed, which simplifies considerably the formulation.

Some aspects of this method have been extensively discussed in the literature, such as in (El Kadiri et al., 2002a; Lewandowsky, 2003). Some of the comments made in the previous reference have been examined in (Beidouri, 2006).

\section{Governing equations}

Figure 1 shows the 2-dof system examined in the present work. It consists of two masses and four spiral springs, with $C_{r}^{l}$ and $C_{r}^{n l}$ being respectively the linear and nonlinear cubic rigidities of the $r^{t h}$ spiral spring. The springs are attached by identical bars of length $l$. These bars are supposed to be mass less, not infinitely rigid, and made of an elastic material which may be slightly deformed. The momentum $\mathcal{M}_{r}$ in the $r^{\text {th }}$ spiral spring is given by :

$$
\mathcal{M}_{r}=-C_{r}^{l} \theta-C_{r}^{n l} \theta^{3}
$$

The undeformed initial position of this system is presented in Figure 1. The two masses $m_{1}$ and $m_{2}$ are excited respectively by two harmonic forces $F_{1}$ and $F_{2}$ chosen in each case according to the mode to be dominately excited (Figure 2) : 


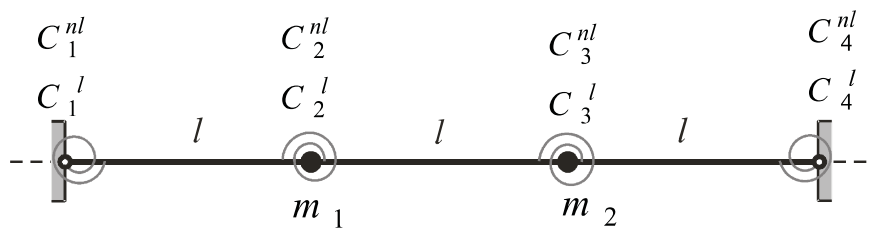

Figure 1. The undeformed initial position of the 2-dof system

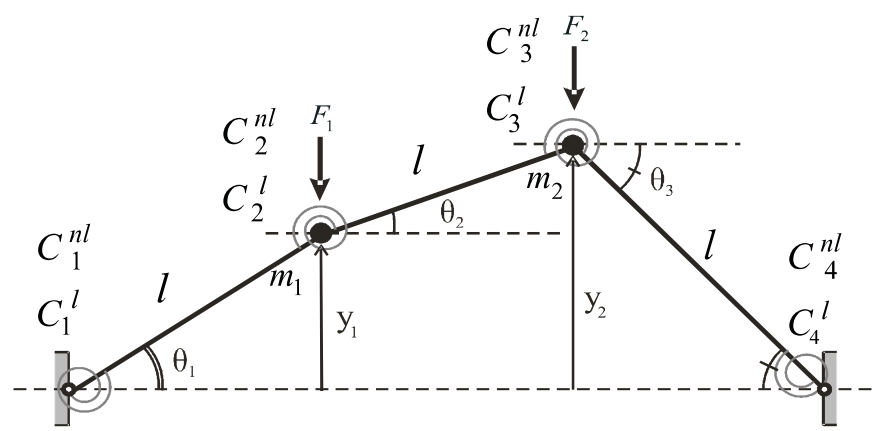

Figure 2. The 2-dof system excited by the two forces $F_{1}$ and $F_{2}$

The displacements of the masses $m_{1}$ and $m_{2}$ from the undeformed positions of the springs are denoted as $y_{1}$ and $y_{2}$. The displacement vector $\{y\}$ defined by $\{y\}^{T}=\left[y_{1} y_{2}\right]$ can be written as :

$$
\begin{aligned}
\{y\} & =y_{1} \vec{u}_{1}+y_{2} \vec{u}_{2} \\
& =\bar{y}_{1} \vec{\phi}_{1}+\bar{y}_{2} \vec{\phi}_{2}
\end{aligned}
$$

where $\left\{\vec{u}_{1}, \vec{u}_{2}\right\}$ is the Displacement Basis (DB) defined by the vectors $\left\{u_{1}\right\}^{T}=\left[\begin{array}{ll}1 & 0\end{array}\right]$ and $\left\{u_{2}\right\}^{T}=\left[\begin{array}{ll}0 & 1\end{array}\right]$ and $\left\{\vec{\phi}_{1}, \vec{\phi}_{2}\right\}$ is the Modal Basis (MB), defined by the vectors $\left\{\phi_{1}\right\}^{T}=\left[\varphi_{11} \varphi_{21}\right]$ and $\left\{\phi_{2}\right\}^{T}=\left[\varphi_{12} \varphi_{22}\right]$ representing respectively the first and second linear mode shapes of the 2-dof system. As can be seen in Equation [2] the components of the displacements of the masses $m_{1}$ and $m_{2}$ in DB and MB are denoted by $\left(y_{1}, y_{2}\right)$ and $\left(\bar{y}_{1}, \bar{y}_{2}\right)$ respectively. The transition matrix from DB to MB is the matrix of the column vectors $\vec{\phi}_{r}$.

The system is supposed to be subjected to a harmonic excitation force given by :

$$
\begin{aligned}
F_{i} & =f_{i} \cos (\omega t) \\
& =\bar{f}_{j} \varphi_{i j} \cos (\omega t) \quad i, j=1,2
\end{aligned}
$$


where $f_{i}$ is the amplitude of the excitation force $F_{i}$ applied to the mass $m_{i}$, expressed in DB, $\bar{f}_{i}$ is the amplitude component of the excitation force $F_{i}$ expressed in MB and $\omega$ is the excitation frequency.

Assuming a harmonic motion defined by :

$$
\begin{aligned}
y_{i} & =A_{i} \cos (\omega t) \\
& =a_{j} \varphi_{i j} \cos (\omega t) \quad i, j=1,2
\end{aligned}
$$

where $A_{i}$ is the modulus of the displacement $y_{i}$ of the mass $m_{i}$ expressed in DB (or the contribution of the normalized vector $\vec{u}_{i}$ of $\mathrm{DB}$ ), and $a_{i}$ is the component of the displacement $y_{i}$ expressed in MB (or the contribution of the normalized vector $\vec{\phi}_{i}$ of $\mathrm{MB})$.

In order to adapt the theory developed in (Beidouri et al., 2008), the kinetic, linear and nonlinear potential energies of the 2-dof system shown in Figure 1 are developed as :

$$
\begin{array}{rlr}
T & =\frac{1}{2} \dot{y}_{i} \dot{y}_{j} m_{i j} & \\
& =\frac{1}{2} a_{i} a_{j} \omega^{2} \bar{m}_{i j} \sin ^{2}(\omega t) & \\
V_{l} & =\frac{1}{2} y_{i} y_{j} k_{i j} & \\
& =\frac{1}{2} a_{i} a_{j} \bar{k}_{i j} \cos ^{2}(\omega t) & \\
V_{n l} & =\frac{1}{2} y_{i} y_{j} y_{k} y_{l} b_{i j k l} & \\
& & \frac{1}{2} a_{i} a_{j} a_{k} a_{l} \bar{b}_{i j k l} \cos ^{4}(\omega t)
\end{array}
$$

where $m_{i j}, k_{i j}$ and $b_{i j k l}$ are respectively the general terms of the mass, the linear rigidity and the nonlinear rigidity tensors in DB, and $\bar{m}_{i j}, \bar{k}_{i j}$, and $\bar{b}_{i j k l}$ are respectively the general terms of the mass, the linear rigidity and the nonlinear rigidity tensors in $\mathrm{MB}$. The relationships between the expressions for these tensors in DB and MB can be obtained as in (Benamar et al., 1993) :

$$
\begin{aligned}
\bar{m}_{i j} & =\varphi_{s i} \varphi_{t j} m_{s t} \\
\bar{k}_{i j} & =\varphi_{s i} \varphi_{t j} k_{s t} \\
\bar{b}_{i j k l} & =\varphi_{s i} \varphi_{t j} \varphi_{p k} \varphi_{q l} b_{s t p q} \\
\bar{f}_{i} & =\varphi_{s i} f_{s} \quad i, j, k, l, s, t, p, q=1,2
\end{aligned}
$$


The kinetic energy of the 2-dof system, exhibiting a harmonic motion, can be written as:

$$
\begin{aligned}
T & =\frac{1}{2} m_{1} \dot{y}_{1}^{2}+\frac{1}{2} m_{2} \dot{y}_{2}^{2} \\
& =\frac{1}{2} \omega^{2}\left(m_{1} A_{1}^{2}+m_{2} A_{2}^{2}\right) \sin ^{2}(\omega t)
\end{aligned}
$$

Identifying Equations [5] and [9] leads to the following expressions for the mass tensor term in DB :

$$
\begin{aligned}
& m_{11}=m_{1} \\
& m_{22}=m_{2} \\
& m_{12}=m_{21}=0
\end{aligned}
$$

For relatively small displacements, compared to the length of the bars, of the discrete system shown in Figure 2, an approximate expression for $\sin \theta_{i}$ can be written as follows :

$$
\sin \theta_{i}=\frac{y_{i}-y_{i-1}}{l} \approx \theta_{i} \quad i=1,3
$$

with $y_{0}=y_{3}=0$, so that the linear potential energy of the 2-dof system can be expressed as :

$$
\begin{aligned}
V_{l} & =\frac{1}{2} \sum_{i=1}^{4} C_{i}^{l}\left(\theta_{i}-\theta_{i-1}\right)^{2} \\
& =\frac{1}{2 l^{2}}\left[\sum_{i=1}^{4} C_{i}^{l}\left(y_{i}-2 y_{i-1}+y_{i-2}\right)^{2}\right]
\end{aligned}
$$

with $y_{-1}=y_{0}=y_{3}=y_{4}=0$ and $\theta_{0}=\theta_{4}=0$.

The generalized linear elastic forces can be obtained by the Equation [13] :

$$
F_{i}^{l}=-\frac{\partial V_{l}}{\partial y_{i}} \quad i=1,2
$$

Applying Equation [13] to the expression for $V_{l}$ given in Equation [12], we get :

$$
\begin{aligned}
& F_{1}^{l}=-\frac{1}{l^{2}}\left[y_{1} C_{1}^{l}-2\left(y_{2}-2 y_{1}\right) C_{2}^{l}+\left(-2 y_{2}+y_{1}\right) C_{3}^{l}\right] \\
& F_{2}^{l}=-\frac{1}{l^{2}}\left[\left(y_{2}-2 y_{1}\right) C_{2}^{l}-2\left(-2 y_{2}+y_{1}\right) C_{3}^{l}+y_{2} C_{4}^{l}\right]
\end{aligned}
$$


On the other hand, by applying Equation [13] to the expression for $V_{l}$ given in Equation [6], we get :

$$
\begin{array}{rlrl}
F_{r}^{l} & =-\frac{1}{2}\left[y_{j} k_{r j}+y_{i} k_{i r}\right] & \\
& =-y_{i} k_{i r} & i, j, r=1,2
\end{array}
$$

in which the classical symmetry relation, i.e. $k_{i j}=k_{j}$, is supposed to be satisfied.

The Equations [14] and [15] permits one to obtain the linear rigidity tensor $k_{i j}$ as follows :

$$
\begin{aligned}
& k_{11}=\frac{1}{l^{2}}\left(C_{1}^{l}+4 C_{2}^{l}+C_{3}^{l}\right) \\
& k_{22}=\frac{1}{l^{2}}\left(C_{2}^{l}+4 C_{3}^{l}+C_{4}^{l}\right) \\
& k_{12}=k_{21}=-\frac{2}{l^{2}}\left(C_{2}^{l}+C_{3}^{l}\right)
\end{aligned}
$$

The nonlinear potential energy of the 2-dof system can be expressed as :

$$
\begin{aligned}
V_{n l} & =\frac{1}{4} \sum_{i=1}^{4} C_{i}^{n l}\left(\theta_{i}-\theta_{i-1}\right)^{4} \\
& =\frac{1}{4 l^{4}}\left[\sum_{i=1}^{4} C_{i}^{n l}\left(y_{i}-2 y_{i-1}+y_{i-2}\right)^{4}\right]
\end{aligned}
$$

with $y_{-1}=y_{0}=y_{3}=y_{4}=0$ and $\theta_{0}=\theta_{4}=0$.

The generalized nonlinear elastic forces can be obtained by Equation [18] :

$$
F_{i}^{n l}=-\frac{\partial V_{n l}}{\partial y_{i}} \quad i=1,2
$$

Applying Equation [18] to the expression for $V_{n l}$ given in Equation [17], we get :

$$
\begin{aligned}
& F_{1}^{n l}=-\frac{1}{l^{4}}\left[y_{1}^{3} C_{1}^{n l}-2\left(y_{2}-2 y_{1}\right)^{3} C_{2}^{n l}+\left(-2 y_{2}+y_{1}\right)^{3} C_{3}^{n l}\right] \\
& F_{2}^{n l}=-\frac{1}{l^{4}}\left[\left(y_{2}-2 y_{1}\right)^{3} C_{2}^{n l}-2\left(-2 y_{2}+y_{1}\right)^{3} C_{3}^{n l}+y_{2}^{3} C_{4}^{n l}\right]
\end{aligned}
$$

On the other hand, by applying Equation [18] to the expression for $V_{n l}$ given in Equation [7], we get :

$$
F_{r}^{n l}=-2 y_{i} y_{j} y_{k} b_{i j k r} \quad i, j, k, r=1,2
$$


in which the symmetry relation ships usually obtained for continuous structures i.e.

$$
\begin{aligned}
& b_{i j k l}=b_{i j l k} \\
& b_{i j k l}=b_{k l i j} \\
& b_{i j k l}=b_{i k j l} \quad i, j, k, l=1,2
\end{aligned}
$$

are assumed to be satisfied in the present model.

The equality of the nonlinear forces, written in Equations [19] and [20] permits one to obtain the nonlinear rigidity tensor $b_{i j k l}$ as follows :

$$
\begin{aligned}
& b_{1111}=\frac{1}{2 l^{4}}\left(C_{1}^{n l}+16 C_{2}^{n l}+C_{3}^{n l}\right) \\
& b_{2222}=\frac{1}{2 l^{4}}\left(C_{2}^{n l}+16 C_{3}^{n l}+C_{4}^{n l}\right) \\
& b_{1122}=b_{2211}=b_{1212}=b_{2121}=b_{1221}=b_{2112}=\frac{2}{l^{4}}\left(C_{2}^{n l}+C_{3}^{n l}\right) \\
& b_{1112}=b_{1121}=b_{1211}=b_{2111}=-\frac{1}{l^{4}}\left(4 C_{2}^{n l}+C_{3}^{n l}\right) \\
& b_{2221}=b_{2212}=b_{2122}=b_{1222}=-\frac{1}{l^{4}}\left(C_{2}^{n l}+4 C_{3}^{n l}\right)
\end{aligned}
$$

\section{Amplitude equation for the nonlinear vibration problem}

\subsection{General equations}

The dynamic behaviour of the system may be obtained by Lagrange's equations for a conservative system :

$$
-\frac{d}{d t}\left(\frac{\partial T}{\partial \dot{q}_{r}}\right)+\frac{\partial T}{\partial q_{r}}-\frac{\partial V}{\partial q_{r}}=F_{r} \quad r=1,2
$$

Replacing $T$ and $V\left(V=V_{l}+V_{n l}\right)$ in this equation by their expressions given above, i.e. Equations [5] to [7], expanding the trigonometric function and applying the harmonic balance method we get the following set of nonlinear algebraic equations :

$$
\frac{3}{2} a_{i} a_{j} a_{k} \bar{b}_{i j k r}+a_{i} \bar{k}_{i r}-a_{i} \omega^{2} \bar{m}_{i r}=\bar{f}_{r} \quad i, j, k, r=1,2
$$

which can be written in matrix form as :

$$
\frac{3}{2}[\bar{B}(a)]\{a\}+[\bar{K}]\{a\}-\omega^{2}[\bar{M}]\{a\}=\{\bar{f}\}
$$




\subsection{The linear solution}

If the nonlinear term $\frac{3}{2}[\bar{B}(a)]\{a\}$ is neglected in Equation [25], the classical eigen value problem, very well known for linear systems is obtained i.e.

$$
[\bar{K}]\{a\}-\omega^{2}[\bar{M}]\{a\}=\{\bar{f}\}
$$

The above equation leads to the classical linear response as follows :

$$
\left\{y\left(a_{1}\right)\right\}_{l}=\frac{\bar{f}_{1}}{\bar{k}_{11}-\omega^{2} \bar{m}_{11}} \vec{\phi}_{1}+\frac{\bar{f}_{2}}{\bar{k}_{22}-\omega^{2} \bar{m}_{22}} \vec{\phi}_{2}
$$

Particularly, if the excitation force is chosen in such a manner that the first mode is exclusively excited, i.e. $\bar{f}_{2}=0$, the response is reduced to :

$$
\left\{y\left(a_{1}\right)\right\}_{l}=\frac{\bar{f}_{1}}{\bar{k}_{11}-\omega^{2} \bar{m}_{11}} \vec{\phi}_{1}
$$

for any amplitude of excitation.

It will be shown below that the situation may be different from this scheme, if the nonlinear effects are taken into account, i.e. the nonlinear term $\frac{3}{2}[\bar{B}(a)]\{a\}$ is not neglected in Equation [25].

\subsection{The nonlinear solution}

Considering now the nonlinear algebraic system (24), it has to be solved for determination of the nonlinear frequency response function of the 2-dof systems treated. This system is solved here using the explicit procedure (First formulation) presented for the first time in (El Kadiri et al., 2002b).

\subsubsection{Solution in the neighbourhood of the first mode}

The 2-dof system is supposed here to be excited in such manner that the first mode is predominant in the response. The so-called first formulation is based on an approximation which consists on assuming, when developping the solution in the neighbourhood of the first nonlinear mode, that the contribution vector $\{a\}^{T}=\left[a_{1} a_{2}\right]$ can be written as $\{a\}^{T}=\left[\begin{array}{ll}a_{1} & \varepsilon_{2}\end{array}\right]$ stating that the second contribution $\varepsilon_{2}$ is very small compared to $a_{1}$. This permits one to simplify the nonlinear algebraic system (24) by neglecting all of the nonlinear terms involving $\varepsilon_{2}$, which leads to :

$$
\begin{aligned}
& \frac{3}{2} a_{1}^{3} \bar{b}_{1111}+a_{1} \bar{k}_{11}-a_{1} \omega^{2} \bar{m}_{11}=\bar{f}_{1} \\
& \left(\bar{k}_{22}-\omega^{2} \bar{m}_{22}\right) \varepsilon_{2}+\frac{3}{2} a_{1}^{3} \bar{b}_{1112}=\bar{f}_{2}
\end{aligned}
$$


Equation [29] allows the following analytical expression for the first contribution $a_{1}$ to be obtained :

$$
a_{1}=\frac{\bar{f}_{1}-\frac{3}{2} a_{1}^{3} \bar{b}_{1111}}{\bar{k}_{11}-\omega^{2} \bar{m}_{11}}
$$

Equation [30] allows an analytical expression for $\varepsilon_{2}$ to be derived, in terms of $a_{1}$ and of the linear and nonlinear dynamic characteristics of the system, i.e.

$$
\varepsilon_{2}=\frac{\bar{f}_{2}-\frac{3}{2} a_{1}^{3} \bar{b}_{1112}}{\bar{k}_{22}-\omega^{2} \bar{m}_{22}}
$$

The nonlinear response of the 2-dof system $\left\{y\left(a_{1}\right)\right\}$ defined in Equation [2] can then be expressed as :

$$
\left\{y\left(a_{1}\right)\right\}=\frac{\bar{f}_{1}-\frac{3}{2} a_{1}^{3} \bar{b}_{1111}}{\bar{k}_{11}-\omega^{2} \bar{m}_{11}} \vec{\phi}_{1}+\frac{\bar{f}_{2}-\frac{3}{2} a_{1}^{3} \bar{b}_{2111}}{\bar{k}_{22}-\omega^{2} \bar{m}_{22}} \vec{\phi}_{2}
$$

The total nonlinear response given by Equation [33] appears as the sum of the linear response given by Equation [27] and the nonlinear term given by the Equation [34] :

$$
\left\{y\left(a_{1}\right)\right\}_{n l}=-\frac{3}{2} a_{1}^{3}\left(\frac{\bar{b}_{1111}}{\bar{k}_{11}-\omega^{2} \bar{m}_{11}} \vec{\phi}_{1}+\frac{\bar{b}_{1112}}{\bar{k}_{22}-\omega^{2} \bar{m}_{22}} \vec{\phi}_{2}\right)
$$

The cubic nonlinear term $a_{1}^{3}$ may be obtained for a given value of the excitation frequency parameter $\omega$ and a given value of the excitation force parameter $\bar{f}_{1}$ via Equation [35] :

$$
\left(\frac{\omega}{\omega_{1 L}}\right)^{2}=1+\frac{3}{2} a_{1}^{2} \frac{\bar{b}_{1111}}{\bar{k}_{11}}-\frac{\bar{f}_{1}}{a_{1} \bar{k}_{11}}
$$

in which $\omega_{1 L}$ is the first linear frequency parameter given by Equation [36] :

$$
\omega_{1 L}=\sqrt{\frac{\bar{k}_{11}}{\bar{m}_{11}}}
$$

\subsubsection{Solution in the neighbourhood of the second mode}

Similar steps are used for determination of the response in the neighbourhood of the second nonlinear mode shape as a function of the contribution $a_{2}$. The 2-dof 
system is supposed here to be excited in such manner that the second mode is predominant in the response. The so-called first formulation is based on an approximation which consists on assuming, when developping the solution in the neighbourhood of the second nonlinear mode, that the contribution vector $\{a\}^{T}=\left[\begin{array}{ll}a_{1} & a_{2}\end{array}\right]$ can be written as $\{a\}^{T}=\left[\begin{array}{ll}\varepsilon_{1} & a_{2}\end{array}\right]$ stating that the first contribution $\varepsilon_{1}$ is very small compared to $a_{2}$. This permits one to simplify the nonlinear algebraic system (24) by neglecting all of the nonlinear terms involving $\varepsilon_{1}$, which leads to :

$$
\begin{aligned}
& \left(\bar{k}_{11}-\omega^{2} \bar{m}_{11}\right) \varepsilon_{1}+\frac{3}{2} a_{2}^{3} \bar{b}_{1222}=\bar{f}_{1} \\
& \frac{3}{2} a_{2}^{3} \bar{b}_{2222}+a_{2} \bar{k}_{22}-a_{2} \omega^{2} \bar{m}_{22}=\bar{f}_{2}
\end{aligned}
$$

Equation [37] allows the following analytical expression for the first contribution $a_{2}$ to be obtained :

$$
a_{2}=\frac{\bar{f}_{2}-\frac{3}{2} a_{2}^{3} \bar{b}_{2222}}{\bar{k}_{22}-\omega^{2} \bar{m}_{22}}
$$

Equation [38] allows an analytical expression for $\varepsilon_{1}$ to be derived, in terms of $a_{2}$ and of the linear and nonlinear dynamic characteristics of the system, i.e.

$$
\varepsilon_{1}=\frac{\bar{f}_{1}-\frac{3}{2} a_{2}^{3} \bar{b}_{2221}}{\bar{k}_{11}-\omega^{2} \bar{m}_{11}}
$$

The nonlinear response of the 2-dof system $\left\{y\left(a_{2}\right)\right\}$ defined in Equation [2] can then be expressed as :

$$
\left\{y\left(a_{2}\right)\right\}=\frac{\bar{f}_{1}-\frac{3}{2} a_{2}^{3} \bar{b}_{1222}}{\bar{k}_{11}-\omega^{2} \bar{m}_{11}} \vec{\phi}_{1}+\frac{\bar{f}_{2}-\frac{3}{2} a_{2}^{3} \bar{b}_{2222}}{\bar{k}_{22}-\omega^{2} \bar{m}_{22}} \vec{\phi}_{2}
$$

The total nonlinear response given by Equation [41] appears as the sum of the linear response given by Equation [27] and the nonlinear term given by the Equation [42] :

$$
\left\{y\left(a_{2}\right)\right\}_{n l}=-\frac{3}{2} a_{2}^{3}\left(\frac{\bar{b}_{1222}}{\bar{k}_{11}-\omega^{2} \bar{m}_{11}} \vec{\phi}_{1}+\frac{\bar{b}_{2222}}{\bar{k}_{22}-\omega^{2} \bar{m}_{22}} \vec{\phi}_{2}\right)
$$

The cubic nonlinear term $a_{2}^{3}$ may be obtained for a given value of the excitation frequency parameter $\omega$ and a given value of the excitation force parameter $\bar{f}_{2}$ via Equation [43] :

$$
\left(\frac{\omega}{\omega_{2 L}}\right)^{2}=1+\frac{3}{2} a_{2}^{2} \frac{\bar{b}_{2222}}{\bar{k}_{22}}-\frac{\bar{f}_{2}}{a_{2} \bar{k}_{22}}
$$


in which $\omega_{2 L}$ is the second linear frequency parameter given by Equation (44) :

$$
\omega_{2 L}=\sqrt{\frac{\bar{k}_{22}}{\bar{m}_{22}}}
$$

\section{Applications}

In this section, some application of the theory presented above are given in order to illustrate the kind of results one can obtain by the present model for various geometries and excitation cases of 2-dof systems, constituted by two masses, and four nonlinear spiral springs.

\subsection{Application 1 : a linear symmetric system (System 1)}

In the first application, a linear symmetric system called system 1, was examined in order to test the model. A symmetric distribution of masses and linear rigidities was adopted. The characteristics of this system are (Figure 3) :

$$
\begin{aligned}
& m_{1}=m_{2}=1 K g, \\
& C_{1}^{l}=C_{2}^{l}=C_{3}^{l}=C_{4}^{l}=10 \mathrm{Nm}, \\
& C_{1}^{n l}=C_{2}^{n l}=C_{3}^{n l}=C_{4}^{n l}=0 \mathrm{Nm}, \\
& l=1 \mathrm{~m} .
\end{aligned}
$$

The excitation amplitude is choosen as follows : $f_{1}=1 N$ and $f_{2}=0 N$.

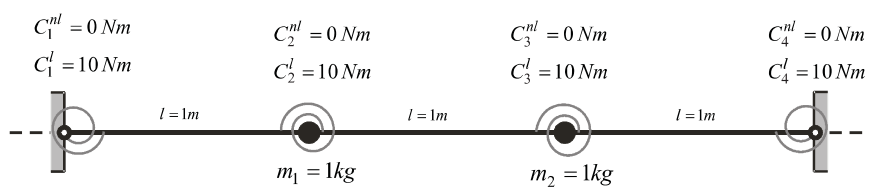

Figure 3. Forced linear symmetric system (System1)

For this first application, the numerical results obtained are plotted in Figure 4(a) in which the displacement amplitudes $A_{1}$ and $A_{2}$ of the masses $m_{1}$ and $M_{2}$ are plotted versus the frequency parameter $\omega / \omega_{1 L}$. In Figure 4(b), the linear frequency response curves are given in terms of the amplitudes of the contributions and versus the frequency parameter in MB. The results corresponding to this excitation corresponds to the classical response in the forced linear model. The curves obtained correspond exactly to the classical response predicted in linear theory (Thompson et al., 1998). 


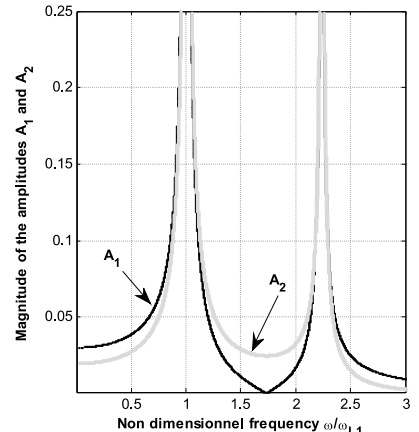

(a)

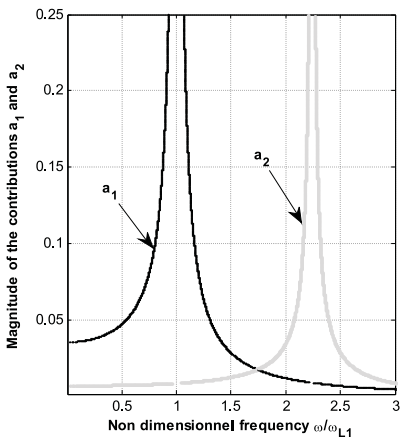

(b)

Figure 4. Linear forced response of system 1 in $D B(a)$ and $M B(b)$

\subsection{Application 2 : a nonlinear symmetric system (System 2)}

In the second application, a nonlinear symmetric system called system 2 , was examined for which a distribution of nonlinear rigidities is adopted. The characteristics of this system are (Figure 5) :

$$
\begin{aligned}
& m_{1}=m_{2}=1 \mathrm{Kg}, \\
& C_{1}^{l}=C_{2}^{l}=C_{3}^{l}=C_{4}^{l}=10 \mathrm{Nm}, \\
& C_{1}^{n l}=C_{2}^{n l}=C_{3}^{n l}=C_{4}^{n l}=150 \mathrm{Nm}, \\
& l=1 \mathrm{~m} .
\end{aligned}
$$

the excitation distribution and amplitude was choosen so that : $f_{1}=1 N$ and $f_{2}=0 N$.

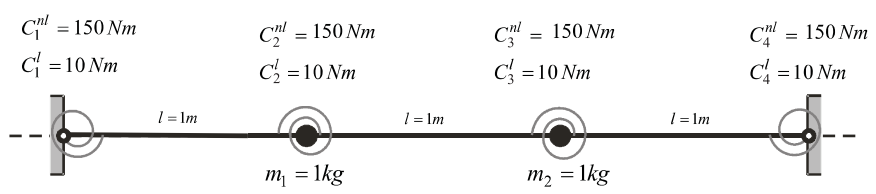

Figure 5. Forced nonlinear symmetric system (System 2)

For the second system shown in Figure 5, calculations have been performed to obtain the nonlinear response in the neighbourhood of the first and second linear mode shapes using Equations [33] to [35] and Equations [41] to [43] in the non dimensional frequency ranges $[0 ; 1,7]$ and $[1,7 ; 5]$ respectively. The numerical results obtained are presented in Figure 6(a) in which the displacement amplitudes $A_{1}$ and $A_{2}$ of the masses $m_{1}$ and $m_{2}$ are plotted versus the frequency parameter $\omega / \omega_{1 L}$. In Figure 6(b), 
the amplitude of the contributions $a_{1}$ and $a_{2}$ in MB are plotted versus the frequency parameter $\omega / \omega_{1 L}$. The frequency response curve obtained here exhibits qualitatively a classical nonlinear behaviour with multivalued regions in which the jump phenomenon could occur. Also, the results corresponding to this excitation show that the nonlinearity observed is of the hardening type with a more accentuated nonlinear behaviour for the second mode, which is the type of behaviour usually observed in geometrically non linear problems (See for example reference (El Kadiri et al., 1999)). Figure 6(c) gives the variation of the displacement amplitude $A_{1}$ as a function of $A_{2}$ and confirms that the two masses are vibrating in phase in the first mode. Figure 6(d) gives the variation of the displacement amplitude $A_{1}$ as a function of $A_{2}$ and confirms that the two masses are vibrating in opposition of phase in the neighbourhood of the second mode.

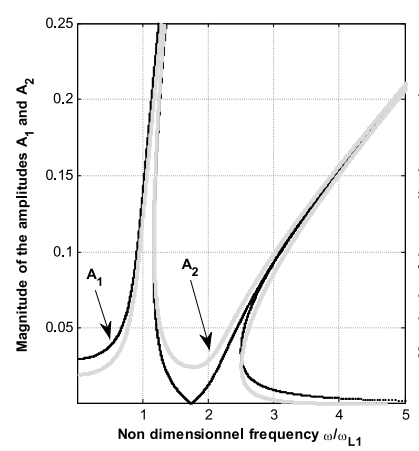

(a)

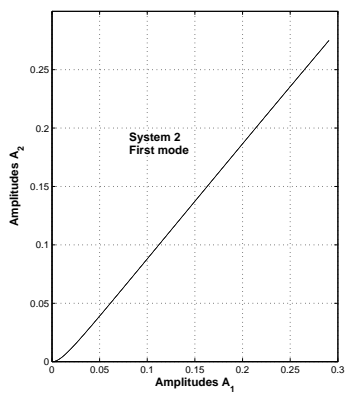

(c)

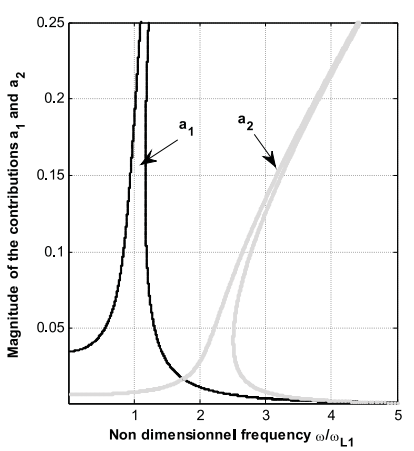

(b)

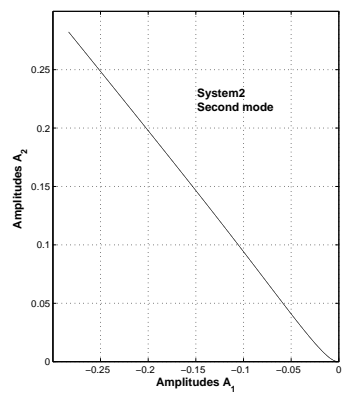

(d)

Figure 6. Nonlinear forced response of system 2 in $D B(a)$ and $M B(b)$. Amplitude $A_{1}$ versus $A_{2}$ of the two masses in the fist mode (c) and second mode (d) 


\subsection{Application 3 : a nonlinear non symmetric system (System 3)}

In this third application, a nonlinear non symmetric system called system 3 is examined, the characteristics of this system are (Figure 7) :

$$
\begin{aligned}
& m_{1}=m_{2}=1 \mathrm{Kg}, \\
& C_{1}^{l}=C_{2}^{l}=C_{3}^{l}=C_{4}^{l}=10 \mathrm{Nm}, \\
& C_{1}^{n l}=C_{2}^{n l}=C_{4}^{n l}=150 \mathrm{Nm} \text { and } C_{3}^{n l}=300 \mathrm{Nm}, \\
& l=1 \mathrm{~m}
\end{aligned}
$$

The excitation distribution is choosen in the two cases considered in order to excite respectively the first mode and the second mode exclusively i.e.

- For the first case : $f_{1}=f_{2}=1 \mathrm{~N}$

- For the second case : $f_{1}=-f_{2}=1 N$

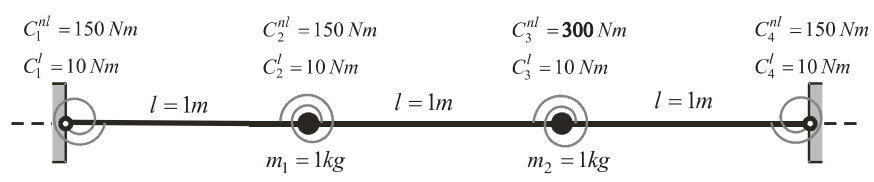

Figure 7. Forced nonlinear non symmetric system (System 3)

\subsubsection{Excitation of system 3 in the neighbourhood of the first mode}

The numerical results obtained when system 3 is excited in neighbourhood of the first mode are presented in Figure 8(a) in which the displacement amplitudes $A_{1}$ and $A_{2}$ of the masses $m_{1}$ and $m_{2}$ are plotted versus the frequency parameter $\omega / \omega_{1 L}$.the result schow a accentuated nonlinear behaviour of the mass 2 . In Figure $8(\mathrm{~b})$, and Figure 8 (c) the nonlinear frequency response curves are given in terms of the amplitudes of the contributions $a_{1}$ and $\varepsilon_{2}$ respectively versus the frequency parameter $\omega / \omega_{1 L}$ in MB. The results corresponding to this excitation show that the nonlinearity observed is of the hardening type and exhibits qualitatively a classical nonlinear behaviour with multivalued regions in which the jump phenomenon could occur. The Figure 8(d) shows that the ratio of the displacement of the two masses is not constant when the amplitude of vibration increases. The variation of the contributions of the two modes with the frequency, shows that the contribution of the second linear mode to the nonlinear response in the neighbourhood of the first mode can be non negligible. The nonlinear response is not proportional to the first mode but involve a contribution of the second mode, due to the nonlinearity. 


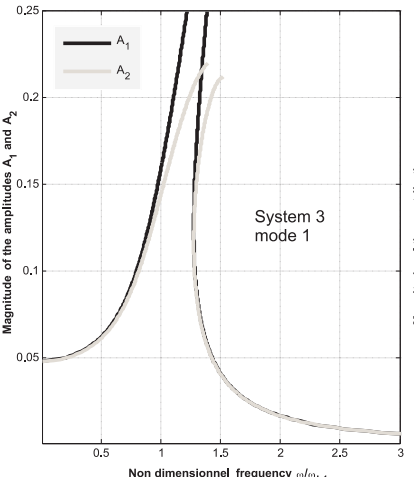

(a)

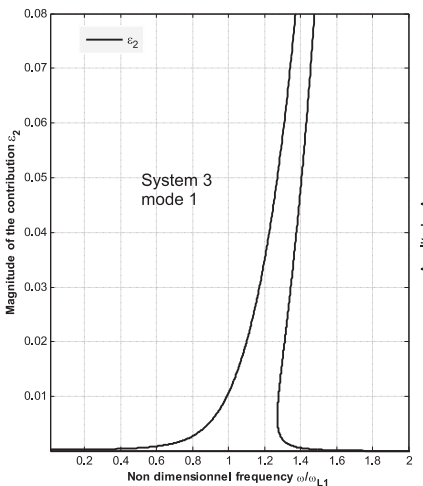

(c)

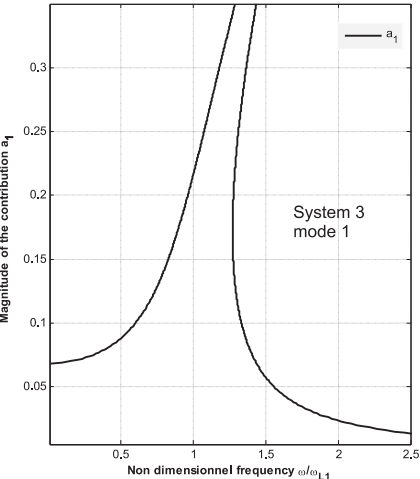

(b)

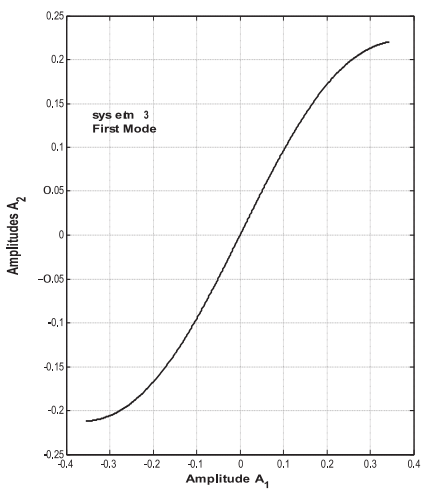

(d)

Figure 8. Magnitude of $A_{1}$ and $A_{2}$ in DB (Mode 1)(a), contribution $a_{1}$ in MB (Mode 1) (b), Contributions $\varepsilon_{2}$, in $M B$ (Mode 1)(c), Amplitudes $A_{2}$ versus $A_{1}$ of the two masses in the fist mode $(d)$

\subsubsection{Excitation of system 3 in neighbourhood of the second mode}

The numerical results obtained when system 3 is excited in the neighbourhood of the second mode are plotted in Figure 9(a) in which the displacement amplitudes $A_{1}$ and $A_{2}$ of the masses $m_{1}$ and $m_{2}$ are plotted versus the frequency parameter $\omega / \omega_{1 L}$. The results schow a much more accentuated nonlinear behaviour of mass 2 . In Figure 9(b), and Figure 9(c). the nonlinear frequency response curves are given in terms of the amplitudes of the contributions $a_{2}$ and $\varepsilon_{1}$ respectively versus the frequency parameter $\omega / \omega_{1 L}$ in MB. A classical nonlinear behaviour, similar to that mentioned above, is observed with multivalued regions in which the jump phenomenon could occur. Also, the results corresponding to this excitation show that the nonlinearity observed is of the hardening type and is more accentuated for the second mode, compared to 
that obtained for the first mode in the above subsection. This type of behaviour agrees qualitatively with that usually observed in geometrically nonlinear vibration problems (See for example reference (El Kadiri et al., 1999)). The Figure 9(d) shows also that the ratio of the displacement of the two masses is not constant when the amplitude of vibration increases. The variation of the contributions of the two modes with the frequency, shows that the contribution of the first linear mode to the nonlinear response in the neighbourhood of the second mode can be non negligible. The nonlinear response is not proportional to the first mode but involve a contribution of the first mode, due to the nonlinearity.

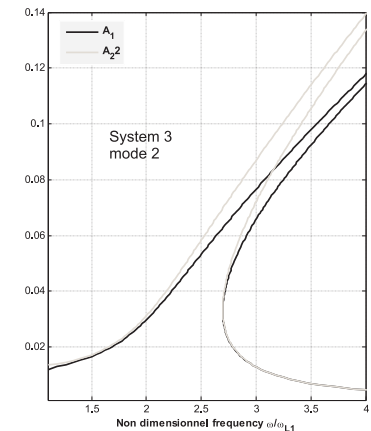

(a)

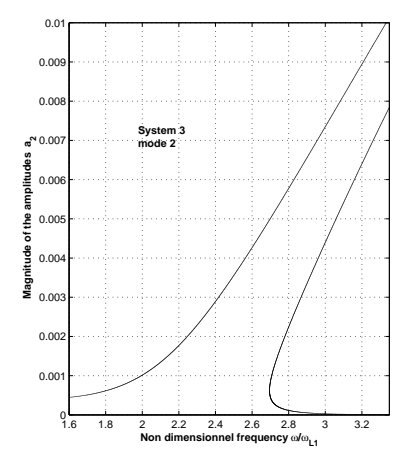

(c)

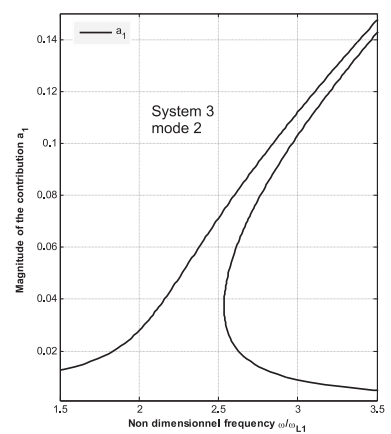

(b)

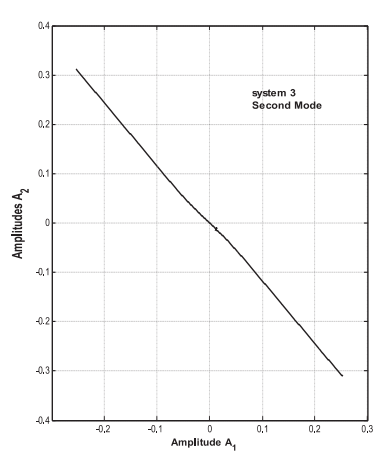

(d)

Figure 9. Magnitude of $A_{1}$ and $A_{2}$ in $D B$ (Mode 2)(a), contribution $a_{2}$ in MB (Mode 2) (b), Contributions $\varepsilon_{1}$, in $M B$ (Mode 2)(c), Amplitudes $A_{2}$ versus $A_{1}$ of the two masses in the second mode $(d)$ 


\section{Conclusion}

The semi-analytical approach to the nonlinear dynamic response of 2 dof systems based on multi-mode analysis has been developed. The applicability of this method to the nonlinear forced vibration of discrete systems with various types of excitations is established. This enabled the nonlinear effect to be taken into account via the fourth order tensor, and the nonlinear frequency dependence on the amplitude to be established. Various types of excitations have been examined. Using the explicit method of solution, the nonlinear amplitude equation of motion has been solved. The nonlinear frequencies and response curves in the neighbourhood of the first and second resonances have been obtained for various types of excitation distributions. The results have shown that the nonlinear response in the neighbourhood of the two linear modes of the 2 dof system considered remains proportional to the corresponding mode in the case of a complete symmetry of the problem. If a non symmetry of nonlinear rigidity is introduced, a clear nonlinear coupling between the two modes appears in the amplitude of vibration. The present formulation and solution is general and simple. It provides a useful tool for studying the nonlinear forced vibration of discrete systems. The method can be readily applied in the study of the large amplitude vibration inducing large curvatures of physically discretized beams. Extensions can be easily made to $\mathrm{N}$ dof systems which may allow an easy treatment of nonlinear forced vibrations of systems exhibiting a localised nonlinearity.

\section{Bibliographie}

Atmani H., Geometrically non-linear dynamic behaviour of isotropic beams hybrid composite and homogeneous plates : New analogical and computer implemented approch to the harmonic distorsion effect and the non linear forced response., Ph.d. thesis, Ecole Mohammadia d'ingénieurs, Rabat, Maroc, 2005.

Atmani H. Seddouki A., Harras B., Benamar R., « A new approach to the geometrically nonlinear dynamic behaviour of a glare 3 hybrid composite panel : Explicit analytical solutions », Colloque International des Problèmes Non linéaires de Mécanique, Metz, France, 25-26 Mai, 2004.

Azrar L., Benamar R., Potier-Ferry M., « An asymptotic-numerical method for large-amplitude free vibrations of thin elastic plates. », Journal of Sound and vibration, vol. 220, n 4, p. 695727, 1999a.

Azrar L. Benamar R., White R. G., « A Semi-analytical approach to the non-linear dynamic response problem S-S and C-C beams at large vibration amplitudes Part I : General theory and application to the single mode approach to free and forced vibration analysis », Journal of Sound and Vibration, vol. 244, n 2, p. 183-207, 1999b.

Azrar L. Benamar R., White R. G., « A Semi-analytical approach to the non-linear dynamic response problem of beams at large vibration amplitudes, Part II : multi-mode approach to steady state forced periodic response », Journal of Sound and Vibration, vol. 255, $\mathrm{n}^{\circ} 1$, p. 1-41, 2002. 
Azrar R., Semi-analytical and asymptotic-numerical methodes for non-lineair vibrations. Application to large amplitude vibrations of beams and plates, Ph.d. thesis, Ecole Mohammadia d'Ingénieurs Rabat, Morocco, 1999.

Beidouri Z., Contribution a une theorie d'analyse modale non-linear. Application aux structures continues et aux systemes discrets a non-linearités localisees, Ph.d. thesis, Ecole Mohammadia d'ingénieurs, Rabat, Maroc, 2006.

Beidouri Z. Eddanguir A., Benamar R., « Geometrically nonlinear free transverse vibration of 2-dof systems with cubic nonlinearities », 7th European conference on Structural Dynamics - Eurodyn (E266), http ://www.isvr.soton.ac.uk/eurodyn2008, 7-9 July, 2008.

Beidouri Z. El Kadiri M., Benamar R., « Geometrically nonlinear transverse vibrations of C-SS-S and C-S-C-S rectangular plates », International Journal of Nonlinear Mechanics, vol. $41, n^{\circ} 1$, p. 57-77, 2006.

Benamar R., Non-linear dynamic behaviour of fully clamped beams and rectangular isotropic and laminated plates, Ph.d. thesis, University of Southampton, England, 1990.

Benamar R. Bennouna M. M. K., White R. G., « The effects of large vibration amplitudes on the fundamental mode shape of thin elastic structures, part I : simply supported and clampedclamped beams. », Journal of Sound and vibration, vol. 2, n 149, p. 179-195, 1991.

Benamar R. Bennouna M. M. K., White R. G., « The effects of large vibration amplitudes on the mode shape and natural frequencies of thin elastic structures, part II : Fully clamped rectangular isotropic plates », Journal of Sound and Vibration, vol. 164, n² 2, p. 295-316, 1993.

Benamar R. Bennouna M. M. K., White R. G., « The effects of large vibration amplitudes on the mode shapes and natural frequencies of thin elastic structures, part III : fully clamped rectangular isotropic plates-measurements of the mode shape amplitude dependence and the spatial distribution of harmonic distortion. », Journal of Sound and vibration, vol. 3, $\mathrm{n}^{\circ} 175$, p. 377-395, 1994.

Bennouna M. M. K., Non-linear dynamic behaviour of a clamped-clamped beam with consideration of fatigue life, Ph.d. thesis, University of Southampton, England, 1982.

Chakraborty G., Mallik A., « Dynamics of a weakly non-linear periodic chain. », International Journal of Non-linear Mechanics., vol. 36, n² 2, p. 375-389, 2001.

Eddanguir A. Beidouri Z., Benamar R., « Geometrically Nonlinear Transverse Vibrations of Discrete Multi-Degrees of Freedom Systems with a Localised Non-Linearity », International Journal of Mathematics and Statistics, vol. 4, n S09, p. 73-87, 2009.

El Bikri K. Benamar R., Bennouna M. K. K., « Geometrically non-linear free vibrations of clamped-clamped beams with an edge crack », Computers and Structures, vol. 84, $\mathrm{n}^{\circ} 7$, p. 485-502, 2006.

El Bikri K. Benamar R., Bennouna M. M. K., « Geometrically non-linear free vibrations of clamped simply supported rectangular plates. Part I : the effects of large vibration amplitudes on the fundamental mode shape. », Computers and Structures., vol. 81, n²0, p. 20292043, 2003.

El Kadiri M. Benamar R., White R. G., « The non-linear free vibration of fully clamped rectangular plates : second non-linear mode for various plate aspect aspect ratios », Sound and vibration, vol. 2, n²28, p. 333-358, 1999.

El Kadiri M. Benamar R., White R. G., « Improvement of the semi-analytical method, based on Hamilton's principle and spectral analysis, for determination of the geometrically non- 
linear free response of thin straight structures. Part I : application to C-C and SS-C beams », Journal of Sound and Vibration, vol. 249, $\mathrm{n}^{\circ}$ 2, p. 263-305, 2002a.

El Kadiri M. Benamar R., White R. G., « Improvement of the semi-analytical method, based on Hamilton's principle and spectral analysis, for determination of the geometrically nonlinear free response of thin straight structures. Part II : First and second nonlinear mode shapes of fully clamped rectangular plates », Journal of Sound and Vibration, vol. 1, n’ 257, p. 19-62, 2002b.

Han W., Petyt M., « Linear vibration analysis of laminated rectangular plates using the hierarchical finite element method- I : Forced vibration analysis. », Computers and Structures, vol. 4, n 61, p. 713-724, 1996a.

Han W., Petyt M., « Linear vibration analysis of laminated rectangular plates using the hierarchical finite element method- I : Free vibration analysis. », Computers and Structures, vol. 4, n 61, p. 705-712, 1996 b.

Harras B. Benamar R., White R. G., « Geometrically non-linear free vibration of fully clamped symmetrically laminated rectangular composite plates. », Journal of Sound and Vibration., vol. $251, n^{\circ} 4$, p. 579-619, 2002.

Haterbouch M. Beidouri Z., Benamar R., « A new simplified approach for solution for geometrically non-linear vibrations of thin circular plates », Proceedings of the Euromech Colloquium 457, on non linear modes of vibrating systems, Frejus, France, 7-9 June, 2004.

Haterbouch M., Benamar R., « The effects of large vibration amplitudes on the axisymmetric mode shapes and natural frequencies of clamped thin isotropic circular plates. Part I : iterative and explicit analytical solution for non-linear transverse vibrations. », Journal of Sound and vibration, vol. 265, $\mathrm{n}^{\circ}$ 1, p. 123-154, 2003.

Haterbouch M. Benamar R., « The effects of large vibration amplitudes on the axisymmetric mode shapes and natural frequencies of clamped thin isotropic circular plates. Part II : iterative and explicit analytical solution for non-linear coupled transverse and in-plane vibrations », Journal of Sound and Vibration, vol. 277, n 1-2, p. 1-30, 2004.

Haterbouch M., Benamar R., « Geometrically non-linear free vibrations of simply supported isotropic thin circular plates. », Journal of Sound and Vibration., vol. 3-5, n 280, p. 903924, 2005.

Jiang D. Pierre C., Shaw S., « Non-linear normal modes for vibratory systems under harmonic excitation. », Journal of sound and vibration., vol. 4-5, n² 288, p. 791-812, 2005.

Kohaupt L., « New upper bounds for free linear and nonlinear vibration systems with applications of the differential calculus of norms. », Applied Mathematical Modelling., vol. 28, $\mathrm{n}^{\circ}$ 4, p. 367-388, 2004.

Lamarque C. H. Janin O., « Modal analysis of mechanical systems with impact non-linearities : Limitation to a modal superposition. », Journal of Sound and Vibration., vol. 4, n 235, p. 567-609, 2000

Lewandowsky R., « Free vibration of strutures with cubic non-linerity-remarks on amplitude and Rayleigh quotient», Computer methods in applied mechanics and engineering, vol. 192, n¹3-14, p. 1681-1709, 2003.

Mei C., « Finite element displacement method for large amplitude free flexural vibrations of beams and plates. », Computers and Structures., vol. 3, n 1, p. 163-174, 1973.

Mei C., « Comments on Lagrange-type formulation for finite element analysis of non-linear beam vibrations. », Journal of Sound and vibration., vol. 94, n 3, p. 445-447, 1984. 
Mei C., « A finite elemnt method for non-linear forced vibrations of rectangular plates. », American Institute of Aeronautics and Astronautics Journal., vol. 7, n 23, p. 1104-1110, 1985.

Mei C., « Discussions of finite element formulations of non-linear beam vibrations. », Сотриters \& Structures., vol. 1, n²2, p. 83-85, 1986.

Moussaoui F., Benamar R., « Authors reply. », Journal of Sound and vibration, vol. 243, n 1, p. 184-189, 2001.

Moussaoui F. Benamar R., White R. G., " The effects of large vibration amplitudes on the mode shapes and natural frequencies of thin elastic shells. Part I : coupled transversecircumferential mode shapes of isotropic circular shells of infinite length. », Journal of Sound and vibration, vol. 5, $\mathrm{n}^{\circ}$ 232, p. 917-943, 2000.

Moussaoui F. Benamar R., White R. G., " The effect of large vibration amplitudes on the mode shapes and natural frequencies of thin elastic shells. Part II : a new approach for free transverse vibration of cylindrical shells of infinite length and of finite length considered at large circumferential wave number », Journal of Sound and Vibration, vol. 255, n 5 , p. 931-963, 2002.

Pesheck E., Pierre C., « A new Galerkin-based approach for accurate non-linear normal modes through invariant manifolds. », Journal of sound and vibration., vol. 5, n² 249, p. 971-993, 2002.

Ribero P., « Multi-modal geometrical non-linear free vibration of fully clamped composite laminated plates. », Journal of Sound and vibration, vol. 1, n²25, p. 127-152, 1999a.

Ribero P., « Non-linear vibration of beams with internal resonance by the hierarchical finiteelement method. », Journal of Sound and vibration, vol. 4, n² 224, p. 591-624, 1999 b.

Ribero P., Petyt M., « Geometrical non-linear, steady, forced, periodic vibration of plates, Part I : Model and convergence studies. », Journal of Sound and vibration, vol. 5, $\mathrm{n}^{\circ} 226, \mathrm{p} .955$ 983, 1999a.

Ribero P., Petyt M., « Geometrical non-linear, steady, forced, periodic vibration of plates, Part II : Stability study and analysis of multi-modal response. », Journal of Sound and vibration, vol. 5, n²26, p. 985-1010, 1999b.

Rosenberg R. M., « The normal modes of non-linear n-Degree-of-Freedom Systems. », Journal of Applied Mechanics., vol. 4, n 29, p. 7-14, 1962.

Rougui M. Moussaoui F., Benamar R., « Non-linear flexural vibrations of thin circular rings. An analytical approach. », Proceedings of the VIII International Conference on Recent Advances in Structural Dynamics., Southampton, UK, 14-16 July, 2003.

Shaw S. W., Pierre C., « Normal modes for non-linear vibratory systems. », Journal of sound and vibration., vol. 164, $\mathrm{n}^{\circ} 1$, p. 85-124, 1993.

Thompson W. T., Dahleh M. D., Theory of vibrations with applications, Editions Prentice-Hall, Upper slide river, new Jersey 07458, USA, 1998.

Vakakis A. F., « Non-linear normal modes (NNMs) and their applications in vibration theory: An overview. », Mechanical Systems and Signal Processing, vol. 11, n 1, p. 3-22, 1997.

White R. G., « Effects of non-linearity due to large deflections in the resonance testing of structures », Sound and vibration, vol. 16, n² 2, p. 255-267, 1971. 